\title{
Eficacia y seguridad del adhesivo tisular Tisuacryl en el cierre de la piel de la episiotomía
}

\author{
*SILVA LEAL, N., *FERNÁNDEZ MASSÓ, J.N., *GÁLVEZ GÓNGORA R.L., \\ *HERRERA VILLENA, J., **MAYELÍN GUERRA, $R$. \\ *Hospital Docente Materno Infantil 10 de Octubre \\ **Centro de Biomateriales de la Universidad de La Habana
}

\begin{abstract}
Resumen
Se realizó un ensayo clínico fase III, en el Hospital Docente Materno Infantil 10 de Octubre, de Ciudad de La Habana, Cuba, entre julio de 1999 y marzo del 2000, con el objetivo de evaluar la eficacia y seguridad del adhesivo tisular Tisuacryl en el cierre de la piel de la episiotomía. Cada grupo constó de 300 pacientes, de acuerdo a los criterios de inclusión establecidos para el ensayo. En el grupo estudio se presentaron menos molestias en la episiorrafia (95.6\%) que en el grupo control (56.3\%) a los 30 minutos post parto, así como al tercer día posterior al nacimiento $(97.6 \%$ vs. $61.3 \%$ ). Al séptimo día, los resultados en el afrontamiento, estética y molestias en la herida fueron significativamente mejores con el adhesivo tisular evaluado. Los riesgos relativos de resultados adversos al séptimo día posterior al parto fueron 3.2 veces mayores en relación con afrontamiento y 2.9 veces con la estética no satisfactorias cuando se utilizó catgut cromado al comparar con el Tisuacryl. Para las molestias en la episiorrafia, el riesgo relativo se elevó a 24.2, todos con diferencias estadísticas significativas. Se concluye que el uso del adhesivo tisular Tisuacryl para el cierre de la piel en la episiotomía tiene menor probabilidad de afrontamiento y estética de la herida no satisfactoria y de menor sensación dolorosa en la herida quirúrgica que el catgut cromado.
\end{abstract}

Palabras claves: adhesivo tisular, Tisuacryl, obstetricia, episiotomía, episiorrafia, ensayo clínico, cirugía.

\section{Introducción}

Los objetivos del cierre quirúrgico de las heridas son ayudar a que los mecanismos de reparación tisular realicen una cicatrización normal y anatómica de los tejidos lesionados y ligar los vasos sanguíneos ${ }^{1,2}$. Para lograr estos propósitos, se utilizan diversos materiales naturales ${ }^{2}$, aunque en las últimas décadas se han desarrollado varios materiales sintéticos, basados fundamentalmente en el cianoacrilato de n-butilo, que se han incorporado al arsenal disponible de materiales que el tiempo ha consagrado $^{3}$, y han sido utilizado extensivamente en variadas especialidades médicas.

De estos últimos se reporta el uso exitoso en el sangramiento de várices esofágicas ${ }^{4-6}$,cierre de

\section{Correspondencia:}

Dra. Norma Silva Leal

Hospital Docente Materno Infantil 10 de Octubre

Facultad de Medicina No. 2, Instituto Superior de

Ciencias Médicas de La Habana

Regla No. 52, esq. a Remedios, Luyanó,

Municipio 10 de Octubre

Ciudad de La Habana, Cuba

E-mail: nsilva@infomed.sld.cu heridas cutáneas ${ }^{7-10}$, cirugía ocular ${ }^{11,12}$, tratamiento de fístulas ${ }^{13,14}$, injertos de piel ${ }^{15}$, tratamiento del varicocele ${ }^{16}$, estomatología ${ }^{17,18}$, cirugía estética facial ${ }^{19,20}$, y otras localizaciones ${ }^{21-23}$.

En obstetricia, antes de la expulsión del feto, es frecuente realizar la episiotomía, intervención que tiene la finalidad de evitar desgarros perineales y vaginales de difícil reparación posterior ${ }^{24}$.

Este proceder se realiza en una zona de gran sensibilidad e irrigación sanguínea, por lo que se produce sangramiento abundante (hasta 500 mililitros se considera como normal la pérdida sanguínea) ${ }^{25}$, y cuya reparación es molesta y dolorosa para la mujer, a pesar de la correcta aplicación de los agentes anestésicos locales.

La reparación tradicional se realiza suturando por planos vagina, tejido muscular, conjuntivo, celular subcutáneo y piel con catgut cromado. El uso en piel de este material en la episiotomía se realiza para evitar la retirada de los puntos en el puerperio, pero tiene el inconveniente que no es adecuado para el cierre de la piel, ya que favorece las infecciones bacterianas, además de producir reacción inflamatoria localmente intensa ${ }^{1}$, con la consiguiente molestia a la madre en esta etapa. 
En el Centro de Biomateriales de la Universidad de La Habana se desarrolló un adhesivo tisular basado fundamentalmente en el cianoacrilato de nbutilo, denominado Tisuacryl, que pasó por la etapa de evaluación preclínica y que tiene registro de aplicación en heridas cutáneas. Actualmente este producto se encuentra en fase de generalización y extensión de sus aplicaciones a varias especialidades médicas, pero no se ha evaluado en la localización objeto de este ensayo.

Con el uso del adhesivo tisular en el cierre de la piel de la episiotomía, se podría aprovechar las ventajas que estos brindan en relación con la sutura clásica: disminución del trauma local, rapidez en la manipulación, disminución de las molestias al paciente y la recuperación más rápida y segura. $\mathrm{Al}$ respecto, en la literatura revisada, se recogen las experiencias de Adoni ${ }^{26}$ y Rogerson ${ }^{27}$, quienes reportaron que con el uso de adhesivo tisular para el cierre de la episiotomía, disminuyeron el dolor y las molestias locales. En este trabajo, nos propusimos evaluar la eficacia y seguridad del adhesivo tisular Tisuacryl en el cierre de la piel de la episiotomía.

\section{Materiales y Métodos}

Se realizó un ensayo clínico controlado Fase II, a partir del $1^{\circ}$ de julio de 1999 y hasta marzo del 2000, en que se completó el muestreo, en el Hospital Docente Materno Infantil 10 de Octubre de Ciudad de La Habana, Cuba. En este hospital se producen alrededor de 4000 partos anuales y es la maternidad con mayor número de nacimientos de la ciudad.

Se realizó la comparación de dos tratamientos:

- Grupo Control: Cierre de la piel de la episiotomía mediante el tratamiento convencional (con catgut cromado 2-0 a puntos simples separados).

- Grupo Estudio: Cierre de la piel con el adhesivo tisular Tisuacryl.

La inclusión en uno u otro grupo se determinó al azar, mediante un listado de números aleatorios generados por computadora. En este se establecía el tratamiento a efectuar en cada paciente que participó en el estudio de acuerdo a los criterios de inclusión detallados más adelante. En el caso de cumplir los criterios de inclusión al ingreso, pero que en la evolución posterior dejó de cumplir alguno, y por lo tanto, salió del estudio, se eliminó de la investigación a la madre del grupo opuesto que le seguía en el listado al efecto.

\section{Selección de los sujetos}

\section{Criterios de inclusión}

1.Primípara con edad al parto entre 20 y 29 años.

2.Ausencia de enfermedades asociadas al embarazo.

3.Trabajo de parto con menos de 8 horas de duración, menos de 3 tactos vaginales desde el ingreso al parto, tiempo de rotura de membranas menor de 6 horas y parto fisiológico (no fórceps ni espátulas). 4.Voluntariedad y consentimiento informado para la participación en el ensayo.

Estos criterios fueron establecidos para tratar de eliminar factores de riesgo de complicaciones en el puerperio, en el período óptimo de la edad reproductiva de la mujer.

\section{Criterios de exclusión}

Quedaron excluidos del estudio todas las pacientes que no cumplieron todos los requisitos de inclusión especificados.

\section{Criterios de salida del estudio y perdidos de vista}

La evaluación para participar en la investigación se efectuó cuando la madre ingresó al servicio de partos de la maternidad, y se realizó por un Especialista en Obstetricia y Ginecología, que comprobó que la gestante cumplía todos los criterios de inclusión para la investigación. Una vez realizada la evaluación, y comprobado que cumplía los requisitos de participación, se procedió a solicitar a la gestante el consentimiento informado para la participación en el ensayo. Se excluyeron del estudio las embarazadas que al ingreso cumplieron los requisitos de inclusión, pero que desde el ingreso al parto dejaron de cumplir al menos uno de los requisitos de inclusión. Como perdidos de vista se consideraron las madres que no se pudieron evaluar a los siete días post parto, aunque es necesario destacar que en este ensayo clínico ninguna gestante estuvo en esta categoría.

\section{Descripción del producto y de los tratamientos}

Nombre del producto y descripción: Tisuacryl, adhesivo tisular. Es un material a base de 2cianoacrilato de n-butilo como componente fundamental. En su composición existe violeta genciana como colorante.

\section{Tratamiento}

Grupo Estudio: Se fijaron y afrontaron los bordes de la piel de la episiotomía con pinzas, y se aplicó el 
adhesivo desde el extremo distal de la herida hacia el extremo vaginal, manteniendo los bordes de la piel unidos durante un minuto.

Grupo Control: La sutura se realizó con puntos de sutura simples separados con catgut cromado 2-0 $\mathrm{y}$ aguja sin filo.

\section{Desarrollo de la investigación y evaluación de la respuesta}

Para la evaluación de la eficacia del adhesivo tisular se comprobó el afrontamiento de los bordes de la piel, que fue realizado de forma independiente e individual por tres investigadores participantes en el ensayo, pero no por el que realizó la aplicación del adhesivo o sutura de la herida. Ninguno de los evaluadores supo la calificación dada por los otros dos, y para obtener la definitiva, se estableció que fuera por mayoría, teniendo en cuenta que solo habían dos categorías de evalución: satisfactoria y no satisfactoria de acuerdo a las variables evaluadas.

Los evaluadores evaluaron el afrontamiento y estética de la herida de acuerdo a su experiencia según:

1.No satisfactorio.

2.Satisfactorio.

Se realizaron tres evaluaciones:

Post - operatorio inmediato: a los 30 minutos inmediatamente posteriores a la culminación del cierre de la piel.

A las 72 horas posteriores al parto.

A los 7 días posteriores al parto.

En relación con las molestias, se preguntó a la paciente sobre las molestias o dolor en la episiorrafia. Para ello se pidió que la evaluara dolorosa o no dolorosa. En este caso, también se definió la evaluación por mayoría.

Se consideró fracaso al tratamiento cuando el adhesivo no logró el cierre de la piel en el acto quirúrgico, y fue necesario utilizar catgut cromado para el cierre de la piel.

\section{Recolección y manejo de los datos}

El llenado de los documentos fue realizado por los investigadores participantes en el ensayo, desde la inclusión del paciente hasta que culminó el seguimiento, y fueron los responsables de la calidad y fidelidad de la información que se recogió. El llenado de los datos se mantuvo actualizado.

\section{Análisis estadístico}

\section{Diseño muestral}

Para el cálculo del tamaño muestral para la comparación de dos proporciones en muestras independientes, se partió del supuesto de que el catgut cromado tiene efectividad del $90 \%$ a los 10 días post parto, según estudio previo realizado en nuestro centro ${ }^{28}$. Para la determinación del tamaño de la muestra se utilizaron tablas estadísticas diseñadas al efecto ${ }^{29}$. Considerando las posibles pérdidas en el seguimiento, que se estimaron del $5 \%$, cada grupo quedó conformado por 300 pacientes.

\section{Análisis estadístico}

Se calcularon las frecuencias relativas de las evaluaciones realizadas, y se contrastaron mediante el test de Chi Cuadrado de Independencia de los Métodos No Paramétricos. El error máximo permisible fue de $\mathrm{p}<0.05$. Se calcularon los riesgos relativos de aparición de efectos adversos en el grupo control, con sus respectivos intervalos de confianza del $95 \%$. Para el análisis de la información, se utilizó el programa Epi-Info 5.0.

\section{Resultados y discusión}

$\mathrm{El}$ afrontamiento a los $30 \mathrm{~min}$. post parto, fue evaluado como satisfactorio en 295 rafias (98.3\%) en el grupo estudio y en 281 (93.6\%) del grupo control. Evaluación no satisfactoria la recibieron 5 madres del primer grupo $(1.7 \%)$ y 19 del segundo $(6.4 \%)$, sin diferencias estadísticas significativas ( $\mathrm{p}>0.05$ ).

Tabla 1. Riesgo Relativo de diferentes resultados adversos en pacientes en las que se utilizó catgut crómico. HDMI 10 de Octubre, julio 1999 - marzo 2000.

\begin{tabular}{|l|c|c|}
\hline & Riesgo Relativo & Intervalo de Confianza 95 \% \\
\hline Afrontamiento No Satisfactorio & 3.2 & $1.1-6.2$ \\
\hline Estética No Satisfactoria & 2.9 & $1.3-9.2$ \\
\hline Molestias en la rafia & 24.2 & $9.1-47.4$ \\
\hline
\end{tabular}

Fuente: Encuesta de la Investigación. 
Estos resultados son similares a los de Gorozpe ${ }^{30}$, que encontró un $2.7 \%$ de dehiscencias de piel de la herida quirúrgica tratadas con adhesivo tisular, aunque en localización diferente a la nuestra. Qureshi ${ }^{31}$ reportó dehiscencias de la herida en el $0.83 \%$ de pacientes tratadas con adhesivo tisular para el cierre de la piel tras laparoscopía.

En relación con la estética de la rafia a los 30 minutos post parto, en las madres en que se utilizó el Tisuacryl, el $97.9 \%$ recibió evaluación satisfactoria, y en las que se utilizó cromado fueron evaluadas satisfactorias el $93.8 \%$, sin diferencias estadísticas ( $\mathrm{p}>0.05)$.

En etapas tan tempranas del proceso de cicatrización, los múltiples mecanismos que en ella intervienen, impiden que se pueda evaluar y predecir adecuadamente cual será la evolución de los tejidos afectados ${ }^{32}$.

A los 30 minutos post parto, el $4.4 \%$ de las pacientes del grupo estudio refirieron que sentían molestia, mientras que en el grupo control esta sensación fue referida por el $43.7 \%$ de las pacientes, con diferencias estadísticas significativas $(\mathrm{p}<0.05)$.

Estos hallazgos son congruentes con la revisión de literatura efectuada ${ }^{22,33,34}$, que coinciden en señalar las ventajas de los adhesivos tisulares sobre las técnicas habituales de cierre con suturas en heridas y pacientes seleccionadas, ya que este material disminuye la respuesta hística inflamatoria local, y por lo tanto, disminuyen también las sensaciones dolorosas percibidas por las pacientes.

El afrontamiento de la rafia a los tres días post parto, no aportó diferencias estadísticas significativas al comparar los resultados ( $p>0.05$ ). Equivalentes resultados se obtuvieron al evaluar la estética de la rafia, ya que la proporción de pacientes fue semejante en ambos grupos (97.6 \% en el primero y $91.0 \%$ en el segundo).

Este resultado está justificado por el hecho de que al tercer día el proceso cicatricial se encuentra en plena etapa inflamatoria ${ }^{27}{ }^{35}$, por lo que el afrontamiento y la estética de la herida guardan más relación con el daño tisular producido que con el material utilizado en la reparación, ya que la influencia de este último es menor que la respuesta del organismo ante la agresión ${ }^{30}$.

$\mathrm{Al}$ evaluar las molestias percibidas por las pacientes, en el grupo estudio, el $97.6 \%$ refirieron no tenerlas, mientras que en el control fue el $61.3 \%$. Estas diferencias entre los grupos evaluados propiciaron significación estadística ( $\mathrm{p}<0.0001$ ), lo que corresponde con los informes de la literatura revisada, que refieren menor frecuencia de dolor y molestias en pacientes en los que se utiliza adhesivo tisular en la reparación de la piel ${ }^{22,25}$.

A partir de los cinco días, comienza la fase proliferativa o fibroblástica de la cicatrización ${ }^{27}$. En esta etapa de consolidación de la reparación tisular, los efectos que provocan los materiales utilizados pueden ser fácilmente evaluados ${ }^{27,30,31}$, ya que disminuyen los efectos del proceso inflamatorio (menos dolor y rubor local) y las reacciones observadas pueden ser evaluadas de una manera más eficaz. Por lo tanto, las posibles diferencias observadas entre los tratamientos deben ser asociadas al mismo y no a los mecanismos intrínsecos de la cicatrización. Por este motivo, se realizó una 3a evaluación a los siete días posteriores al parto.

En relación con el afrontamiento, se observó diferencia significativa $(p<0.05)$, ya que en el grupo estudio fue satisfactorio en el $94.3 \%$ de ellas, y en las que se utilizó catgut, esta proporción fue de $80.6 \%$.

Al respecto se señala ${ }^{25,26}$ que los adhesivos tisulares pueden ser usados de forma satisfactoria y efectiva en el cierre de las heridas de la piel, y es un método seguro y eficiente, con poca morbilidad y resultados satisfactorios a largo plazo sobre la estética de la herida quirúrgica.

$\mathrm{Al}$ evaluar la estética, fueron satisfactorias 282 madres del grupo estudio (94.0\%), y en el grupo control 248, para el $81.6 \%$ ( $\mathrm{p}<0.05$ ). En relación con las molestias percibidas en la rafia, en el grupo estudio percibieron molestias 7 madres (2.4\%), y en el grupo control este resultado lo reportaron 167 puérperas, para el $56.7 \%$ de las componentes de este grupo. Estas diferencias observadas propiciaron significación estadística elevada ( $\mathrm{p}<0.0001)$ a favor del producto evaluado.

Al utilizar catgut cromado (tabla 1), el riesgo de afrontamiento no satisfactorio al séptimo día es 3.2 más elevado (IC $95 \% 1.1$ - 6.4), el de estética no satisfactoria 2.9 veces (IC $95 \% 1.3$ - 9.2), y el de molestias en la rafia 28.2 veces (IC $95 \% 9.1$ 47.4) mayor que con el uso del adhesivo tisular evaluado.

En relación con las reacciones adversas, no presentaron reacciones al producto ni en las pacientes ni en los operadores del mismo. Tampoco se reportaron fracasos.

\section{Biliografía}

1. Sanz LE: Wound management- matching materials and methods for best results, Contemporary $\mathrm{Ob} /$ Gyn, 30(5):86-114, 1987.

2. Stone JK: Materiales de sutura, Clin Obstet Gynecol, 3: 687-692, 1988. 
3. Hatko WJ, Ghanekar G, Kemmann E Suture materials currently used in obstetric gynecology surgery in the United States: questionnaire survey. Obstet Gynecol, 163: 448, 1982.

4. Lo GH, Lai KH, Cheng JS, Hwu JH y cols: A prospective, randomised trial of sclerotherapy versus ligation in the management of bleeding oesophageal varices, Hepatology, 222(2): 466-471, 1995.

5. Feretis C, Dimopoulus C, Benakis $\mathbf{P}$, Kaliakmanis B y cols: N-butyl-2cianoarilate (Histoacryl)plus sclerotherapy versus sclerotherapy alone in the treatment of bleeding esophageal varices: a randomized prospective study. Endoscopy, 27(5): 355-357, 1995.

6. Kok KYY, Kum CK, Goh PMY: Endoscopic hemostasis of upper gastrointestinal bleeding with histoacryl: laste resort before surgery, Endoscopy, 28(2): 256-258, 1996.

7. Knight J.: Proper use of skin adhesives for wound repair, J Am Acad Nurse Pract 2001 Jan;13(1):13-4.

8. Eaglstein WH, Sullivan TP, Giordano PA, Miskin BM.: A liquid adhesive bandage for the treatment of minor cuts and abrasions, Dermatol Surg 2002 Mar;28(3):263-7.

9. Singer AJ, Quinn JV, Clark RE, Hollander JE: Closure of lacerations and incisions with octylcyanoacrylate: a multicenter randomized controlled trial, Surgery 2002 Mar;131(3):270-6.

10. Stagnitti F, Schillaci F, De Pascalis M, Mongardini M, Patrizi V, Cilurso F, Costantini A, Dall'Olio D, Priore F, Tiberi R, Natalini E: Use of adhesives in emergency surgery, G Chir 2001 Aug-Sep;22(89):309-12.

11. Markowitz GD, Orlin SE, Frayer WC y cols: Corneal endothelial polymerization of histoacryl adhesive, Ophthalmic Surg, 26(3): 256-258, 1995.

12. Veloudios A, Kratki V, Heatcote JG y cols : Cyanocrilate tissue adhesive in blepharoplasty, Ophtalmic Plast Reconstr Surg, 12(2): 89-97, 1996.

13. Drury AE, Grundy A : Management of oesophageal fistula by by radiologically-guided instillation of tissue adhesive, Clin Radiol, 50(5): 335-338, 1995.

14. Gutiérrez C, Barrios JE, Lluna J y cols : Recurrent tracheoesophageal fistula treated with fibrin glue, J Pediatr Surg, 29(12): 1567-1569, 1994.

15. Zaki I, Scerri L, Millard L: Split skin grafting on severely damaged skin: a technique using absorbable tissue adhesive, J Dermatol Surg Oncol, 20(12): 827-829, 1994.

16. Comhaire E, Zalata A, Schoonjans E Varicocele: indications for treatment, Int J Androl, 18(Supp 2): 67-71, 1995.

17. Takeuchi M, Hayashi S, Minemoto I: US Patent No. 4180913, 19800101.

18. Berkman M, Cuccolor F, Levin $\mathbf{N}$ y cols: Pulpal response to isobutyl cyanocrilate in human teeth, $\mathrm{J}$ Amer Dent Assoc, 8(3): 140, 1971.
19. Kamer FM, Joseph JH: Histiacryl: its use in aesthetic facial plastic surgery, Arch Otolaryngol Head Neck Surg, 115: 193-197, 1989.

20. Rojas WE, Di Martino E, Harandi B, Westhofen M.: Long-term results of suture-free cutanous wound closure in head and neck incisions with octylcyanoacrylate topical skin adhesive, Laryngorhinootologie 2002 Sep;81(9):644-8.

21. Szyllo K, Wlodarczyk B, Krekora M, Lewy J, Oszukowski P, Wilczynski J.: Application of skin glue in wound repair after laparoscopy, Ginekol Pol 2002 Jun;73(6):567-70.

22. Shamiyeh A, Schrenk P, Stelzer T, Wayand WU: Prospective randomized blind controlled trial comparing sutures, tape, and octylcyanoacrylate tissue adhesive for skin closure after phlebectomy, Dermatol Surg 2001 Oct;27(10):877-80.

23. Currie LJ, Sharpe JR, Martin R: The use of fibrin glue in skin grafts and tissue-engineered skin replacements: a review, Plast Reconstr Surg 2001 Nov;108(6):1713-26.

24. Rigol O: Obstetricia y Ginecología, Tomo I, páginas 130-134, Ediciones Revolucionarias, La Habana, 1983.

25. Botella J, Clavero JA: Tratado de Ginecología, Ediciones Revolucionarias, La Habana, 1983.

26. Adoni A, Anteby $\mathbf{E}$ The use of Histoacryl for episiotomy repair, Br J Obstet Gynecol, 98(5): 476478, 1991.

27. Rogerson L, Mason GC, Roberts AC: Preliminary experience with twenty perineal repairs using Indermil tissue adhesive, Eur J Obstet Gynecol Reprod Biol 2000 Feb;88(2):139-42.

28. Martínez A: Alta oportuna de madres y recién nacidos de bajo riesgo, Trabajo para optar por el Título de Especialista de Primer Grado en Ginecología y Obstetricia, Hospital Docente Materno Infantil 10 de Octubre , 1997.

29. Lwanga SK, Lemeshow $\mathbf{S}$ : Sample size determinations in health studies. A Practical Manual, World Health Organization, Geneva, pag. 35, 1991.

30. Gorozpe C, González J, Santoyo S. Closure of the skin with cyanoacrilate in surgical wounds after tubal ligation, Ginecol Obstet Mex, 65:64-67, 1997.

31. Qureshi A, Drew P, Duthie G: n-butyl cyanoacrilate aghesive for skin closure of abdominal wounds: preliminary results. Ann R Coll Surg Engl, 79(6):414415, 1997.

32. Rodríguez HN: Cirugía Ginecológica y Obstétrica. Ed. Pueblo y Educación, Tomo I, pág. 53, Ciudad de La Habana, Cuba 1993.

33. Giray CB, Atasaver A, Durgun B, Araz Z: Clinical and electron microscope comparison of sutures and n-butyl-cyanoacrilate in human mucosa. Aust Dent J, 42(4): 255-258, 1997.

34. Singer AJ, Hollander JE, Valentine SM, Turque TW, McCuskey CF: Prospective, randomized, 
controlled trial of tissue adhesive (2-cyanoacrilate) vs. Standard wound techniques for laceration repair. Acad Emerg Med, 5(2): 94-99, 1998.
35. Sanz LE: Wound management - matching materials and methods for best results, Contemporary $\mathrm{Ob} /$ Gyn, 30(5):86-114, 1987. 